Proceedings of the 2011 Winter Simulation Conference

S. Jain, R.R. Creasey, J. Himmelspach, K.P. White, and M. Fu, eds.

\title{
AGENTIZING THE SOCIAL SCIENCE OF CRIME
}

\author{
Steven P. Wilcox \\ Serco Inc. \\ 1818 Library Street \#1000 \\ Reston, VA 20190, USA
}

\begin{abstract}
Using the subject matter of neighborhood crime, we explore how to conduct conventional quantitative social science using simulation as a way of formulating theory and performing empirical tests of theory, thus replacing the dominant methodological paradigm. Here we simulate in abstract form a complete system of social relationships to reflect applicable social theory, which in many cases takes the form of prose. This requires integrating the theories and adding implied elements to make a coherent, parameterized system. The next step is calibrating the model to measures of the type that would be normally employed to test the relevant theories. Re-implementing Wilcox's (2005) Matlab-based crime model in Java and exploring model variations, we find patterns in the simulation outputs that highlight the potential difficulties of matching a published correlation matrix and are reminded that simulation modeling is more exacting than social science argument stated as prose and based on group-level conceptual constructs.
\end{abstract}

\section{INTRODUCTION}

How should one conduct a general program of social science using simulation models as its representation of social theory and quantitative methodology? Fully doing so requires methodological advances in representing social phenomena comparably to extant social theory as well as meeting the twin challenges of model validation and parameter estimation needed for comparability to statistical quantitative methodologies. Here we examine how these issues play out in secondary analysis of data on violent crime using an agent-based simulation model, which illustrates one way of performing the simulation and its attendant difficulties, including the need to achieve model validation before models can be statistically tested.

Many have noted the discursive nature of sociological argument, either as a good thing (Sica 2004) or a shortcoming (Mahoney 2004). Simulation modeling in the form of computational social science is emerging as an alternative paradigm, but it is time to go beyond demonstrations of promise and develop agent-based modeling as an alternative methodology on an equal basis to the traditional paradigms. In this paper we examine how these issues play out in the case of the examination of neighborhood crime by Browning, Feinberg, and Dietz (2004).

In utilizing quantitative social science methodology in domains such as neighborhood crime, a number of important difficulties are encountered. Deriving hypotheses from theories suffers the difficulty that the theories at issue are often not formal deductive systems, or may be a stylized model that leaves out important elements. Moreover, arriving at hypotheses is complex due to the necessary use of nonexperimental data, the existence of many different, interacting processes and actors, and the inability to observe of key elements such as the motivations of actors.

Many of these difficulties are evident in the subject matter of neighborhood crime. In this paper we work with a one-dimensional conception of crime and social theories of it that may be more apt in the ghetto than a corporate boardroom. Social theory posits that the rate of crime is influenced by reciprocal 
social influence in attitudes and behavior, much of which is unavailable for measurement by social scientists although these may be conceptualized as social network phenomena. Another factor is the level of social disorganization in the community, which, while not a clearly defined concept, is affected by such factors as the rate of residential migration.

In view of these difficulties, agent-based modeling may aid in the performance of more rigorous sociology by providing a formal system of reasoning for the purpose of deriving empirical hypotheses. While Mahoney (2004) calls for actual agents and actors in the theory of historical sociology, that does not end the discussion. Here we will attempt to use an agent-based model as a methodological improvement over the theoretical arguments and methodological approach of the subject paper, in which it is desired to substantiate a view of crime as occurring within a context of a network of social exchange relationships that tend to impede the suppression of crime.

\section{MODELING SOCIAL THEORIES OF CRIME}

Sociological theories of crime are based on hypotheses about social interactions in a social network such as a neighborhood. On the other hand there are economic theories of crime, such as rational choice theory (Becker 1968) and the conception of peer effects as positive and negative externalities (Glaeser, Sacerdote, and Scheinkman 1996; Calvó-Armengol and Zenou 2004), which are also relevant to building a simulation model that covers competing perspectives.

Social disorganization theory (Shaw and McKay 1969; Kasarda and Janowitz 1974; Kornhauser 1978) views interpersonal social attachment as a good thing. According to Shaw and McKay, poverty, residential instability, and ethnic heterogeneity promote crime by inhibiting the formation of neighborly networks and attenuating community-level action against crime. According to Kasarda and Janowitz, extensive friendship and kinship bonds strengthen neighborhood attachment, and Kornhauser finds that weak bonds mediate the effect of disadvantage on the capacity for social control.

The cultural transmission model (Whyte 1937; Wilson 1996; Crane 1991) focuses on the legitimate social networks as bulwarks against a counter-culture of crime. The criminal subculture emerges in opposition to mainstream culture, and strong networks in socially disadvantaged communities may facilitate its spread. Thus there is a contagion of problem behaviors, for which gang culture provides social support.

Browning, Feinberg, and Dietz (2004) propose and empirically support a negotiated co-existence model, in which social networks are a source of general social capital for offenders, which tends to protect them. Therefore the attitude of neighborly efficacy to fight crime tends towards the suppression criminal behavior, but is offset to some degree by social capital. Thus social disorganization theory is not quite right, but Browning, Feinberg, and Dietz wish to avoid attributing too much organizational capacity to the criminal networks as well.

The economic literature on peer effects in crime is intriguing as a contrast because it is inherently agent-oriented. Becker (1968) looks at crime from a rational choice perspective. Following this perspective, Makowsky (2006) looks at the effect of population shocks over time with an agent-based model. Looking at the puzzle of the variability of crime rates across time and space, the analyses by Glaeser, Sacerdote, and Scheinkman (1996) and Calvó-Armengol and Zenou, (2004) highlight the importance of heterogeneity in agents' toleration for crime as a moderator of peer influence effects, which act as source of training and facilitation. The positive externalities due to the interactions between criminals contrasts with their aggregate competition for resources.

The model by Glaeser, Sacerdote, and Scheinkman (1996) is intriguing in that it employs a circular social network and three types of social actors, such as in Figure 1. The model described here incorporates similar modeling ideas, but mapped into a flat, two-dimensional terrain as this is more like a physical neighborhood.

As a step towards the encapsulation of the theories of neighborhood crime into an agent-based model, observe their depiction in a combined path diagram in Figure 2. As an indication of the complexity of the subject, note that all the arrows go from the self to the other as well as the other way around. For presentational clarity, the symmetrical arrows are not presented in the case of the ecological parameters. Anoth- 


\section{Wilcox}

er indication of the complexity of the subject, note that there are ecological variables (blue) as well as individual-level variables (green) and exogenous parameters and endogenous personal traits that must be put in relation to each other.

The interaction posited by Browning, Feinberg, and Dietz (2004) in which a social exchange relationship with the other impeded the benefit of anti-crime efficacy, is given by the red arrows and the yellow circles. The arrows for the social disorganization theory (magenta) are redundant as they also cover the effect of efficacy on the other's criminality. As a token of the rational choice theory, the effects of disadvantage and density on one's criminal propensity are accounted for by the green arrows, and they are present in the model of Browning, Feinberg, and Dietz. The cultural transmission paradigm is covered by the arrows from relationship to the other's criminality, and from criminality to criminality.

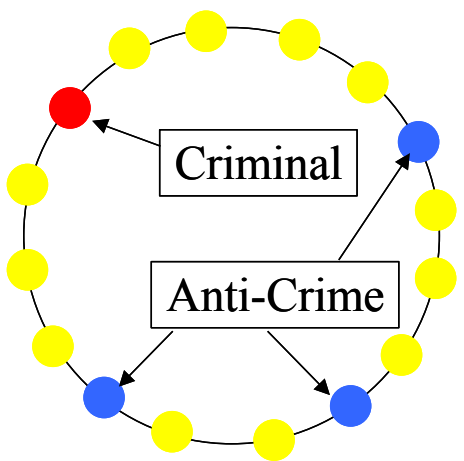

Figure 1: The social network Glaeser, Sacerdote, and Scheinkman (1996) employ to model crime. This represents an agent-based model of social influence on the circular network with variability in the susceptibility to social influence playing an important role in the results.

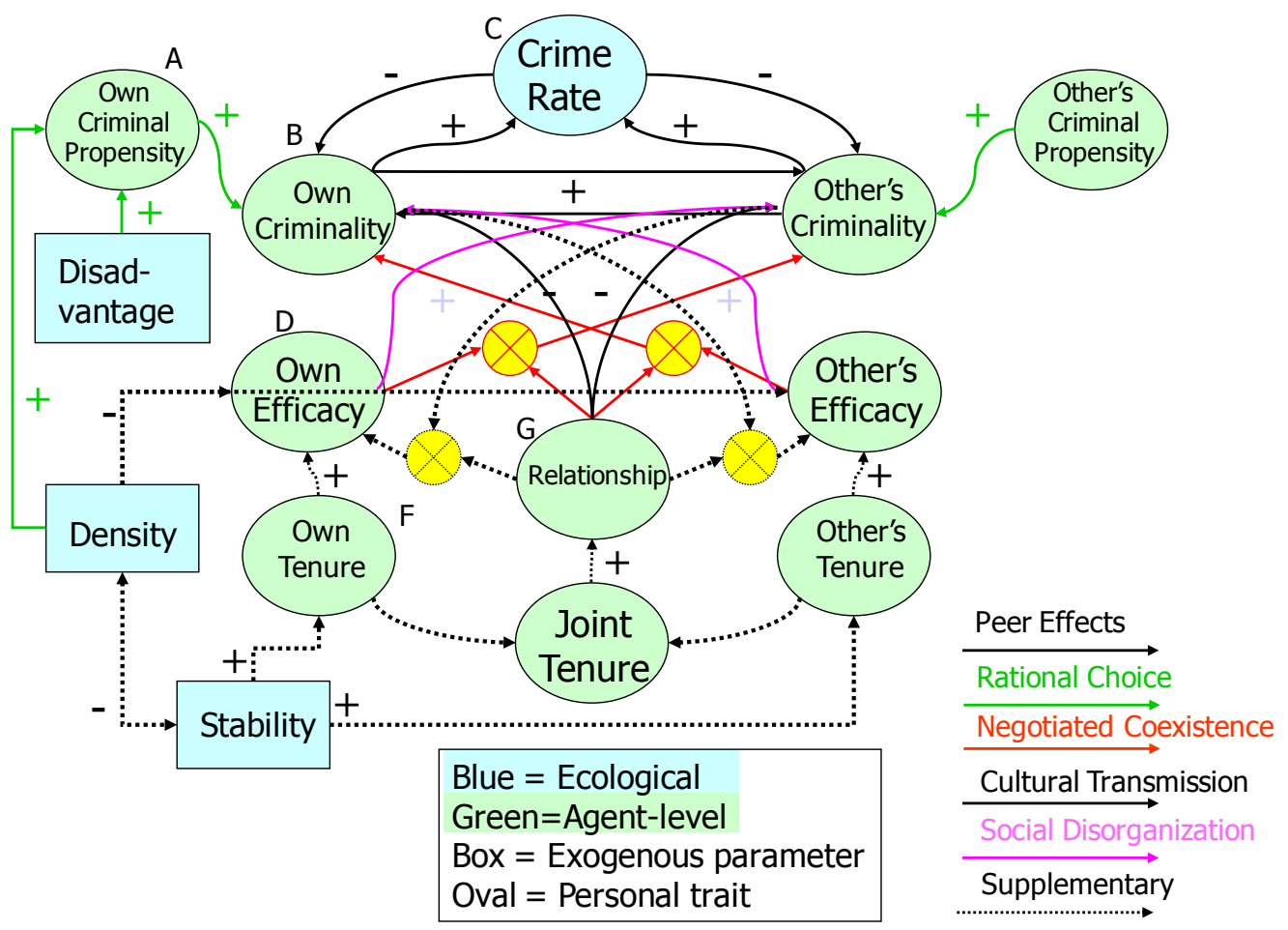

Figure 2: Interrelation of Simulation Elements to Themselves and Social Theories 
To the sociological theories has been added the basics of the peer effects literature from economics (Glaeser, Sacerdote, and Scheinkman 1996; Calvó-Armengol and Zenou 2004), in which heterogeneity of agents, synergy between associated criminals, and competition for scarce resources are important features. These numerous theories notwithstanding, it is also necessary to consider supplementary perspectives. The other's criminality may affect one's own efficacy to fight crime, and it may be argued from cognitive consistency theory that anything capable of affecting behavior may also affect attitudes, and that behavior also affects attitudes.

In the mapping of simulation elements in Figure 2, there are features that present a challenge for identifying parameter values from actual empirical data. Note in particular the embedded loop between an actor's criminality and the other's efficacy. This creates circular causal loops throughout the social system, as depicted in Figure 3.

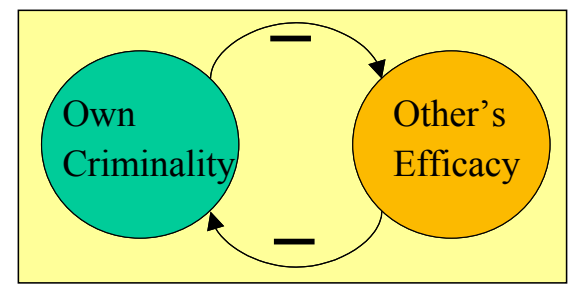

Figure 3: An Identification Issue

\section{AGENT-BASED MODEL DEVELOPMENT}

\subsection{Overview}

The Matlab-based model of crime utilized in Wilcox (2005) works on two levels: interpersonal influence on a social network, such as in Harrison and Carroll (2002) and interaction with the social/economic environment. To integrate the sociological and economic theories of crime, the crime model incorporates a two-dimensional analogue of the circular social influence network considered by Glaeser, Sacerdote, and Scheinkman (1996), in which the features of non-homogeneous occupational preferences and competition among criminals for scarce economic resources create a situation in which disparate equilibria are possible and the crime rate can vary significantly over time. In addition, it includes the interplay between the attitude of neighborly anti-crime efficacy and the behavior of being a criminal.

Since neighborhoods are geographical, this model represents the attitudes and behavior of residents on a 2-D 50 by 50 lattice, but using the toroidal topology to avoid edge effects. In a real neighborhood, one has more than the eight neighbors present in many lattice models. Here we use the Moore neighborhood of radius 4, giving a set of 80 neighbors an agent may form friendships with and be influenced by. However, the crime rate's economic effect on occupational choice is taken over the entire set of 2500 agents.

In this model, agents may be either criminals or law abiding, and they possess a level of "efficacy". Their underlying criminal propensities are heterogeneous within the neighborhood. Social influence is conveyed at three levels: friends, non-friends in the Moore neighborhood, and the community as a whole. Agents may move out of the neighborhood, and thus be immediately replaced. They also may change between the status of criminal and non-criminal, and form friends. All friendships are two-way.

\subsection{Path Model and Parameterization}

The path diagram in Figure 4 describing the actual simulation differs from the path diagram describing the related theories in that it resolves the redundancies among the theories considered. In addition it resolves questions such as whether it makes sense to apply an agent's attitude of efficacy (against crime) if that individual is a criminal. 


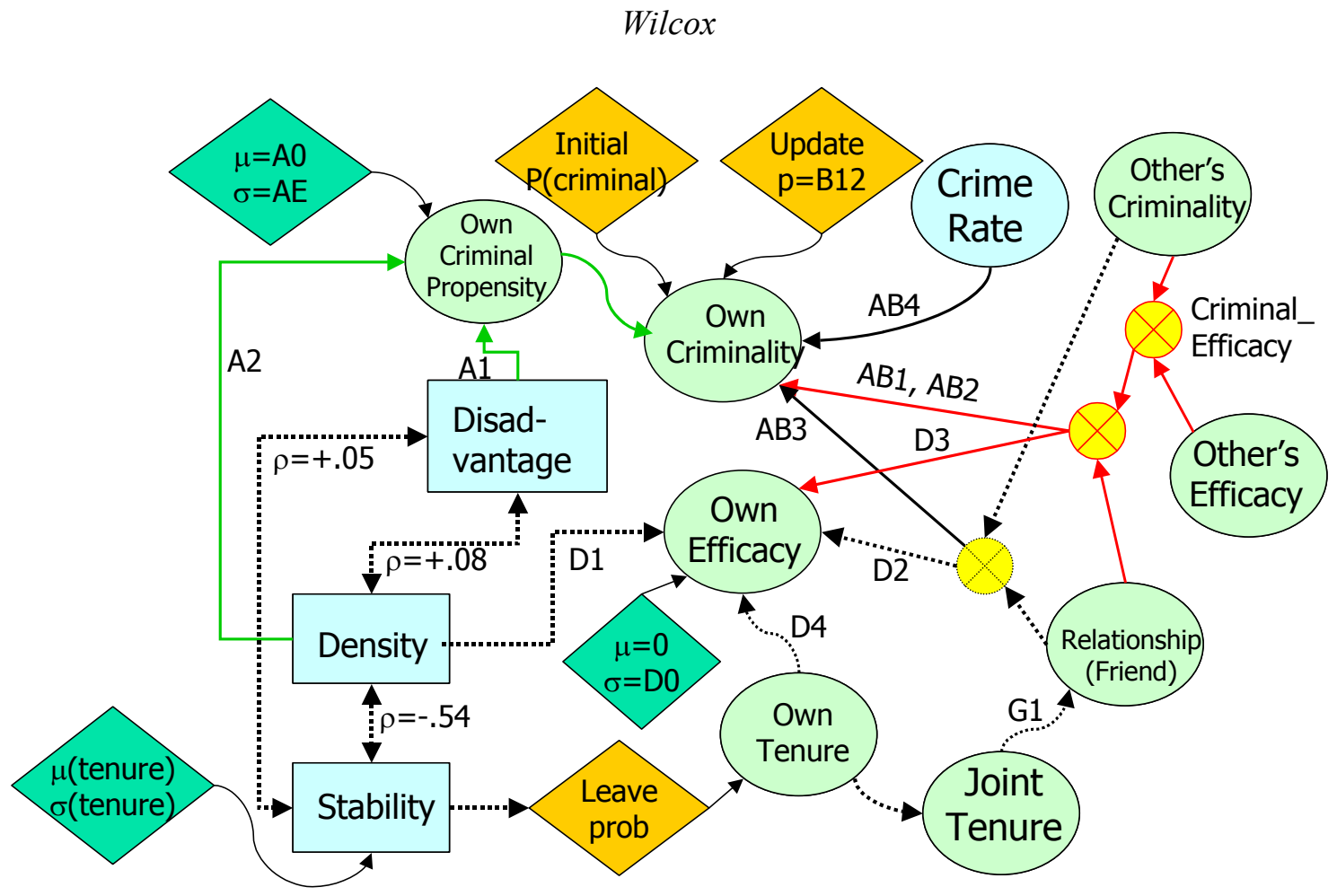

Figure 4: Path model describing the dynamic relationship between the conceptual variables as implemented in the simulation model.

Overall, nineteen parameters corresponding to the path model describe the simulation process. In the blue boxes, Density, Stability, and Disadvantage are unit-free exogenous variables that drive residents' criminal propensities, efficacy, and probability of leaving, seen in parameters $A 1, A 2$, and $D 1$. In the green diamonds are scale and location parameters related to these exogenous variables. Tenure is influenced by a yearly leaving probability that is derived from the stability parameter. Criminality is a choice that is initialized and then reviewed with probability $B 12$. If it is to be updated, the agent is designated a criminal according to a criminal propensity, which is heterogeneous between agents, as well as the influences of friends and neighbors. In accordance with the peer effects literature, the global crime rate has a suppressing effect $(A B 4)$ on an individual's propensity to choose criminality, while friends who are criminals have a separate effect ( $A B 3)$. The value of efficacy used in the influence calculations depends on whether the person doing the influencing is a criminal, in which case the Criminal_Efficacy parameter is used. The effective efficacy of the other thus computed has two different effects (with linear coefficients $A B 1$ and $A B 2$ ) on the other's occupational choice, depending on whether the other is a friend or just a neighbor. The update of Efficacy is affected by the crime rate among the agent's friends according to coefficient D2, and their Efficacy, according to coefficient D3. Efficacy is also influenced by tenure (D4) and density $(D 1)$. Finally, friendships grow with joint tenure, according to probability $G 1$ each year.

One may notice the model's complexity. The path model implied by theory is fairly complex, yet incomplete, and yet things had to be added. Effect of time on relationship building is common sense, but not explicitly stated as theory. Cognitive consistency theories could be further exploited to suggest additional relationships between one's own attitudes and own behavior.

\subsection{Agent-Based Lattice Architecture}

While many agent-based models posit actors on a torroidal lattice, the usual pattern is to assume interacttion occurs when actors are adjacent such as Schelling's (1971) Model of Segregation or Lustick's ABIR (2000) and PS-I (2002) models of political influence. As depicted in Figure 5, a 50 by 50 lattice is chosen 


\section{Wilcox}

as comparable in size to a neighborhood, but neighbors are counted within a Moore neighborhood of radius four.

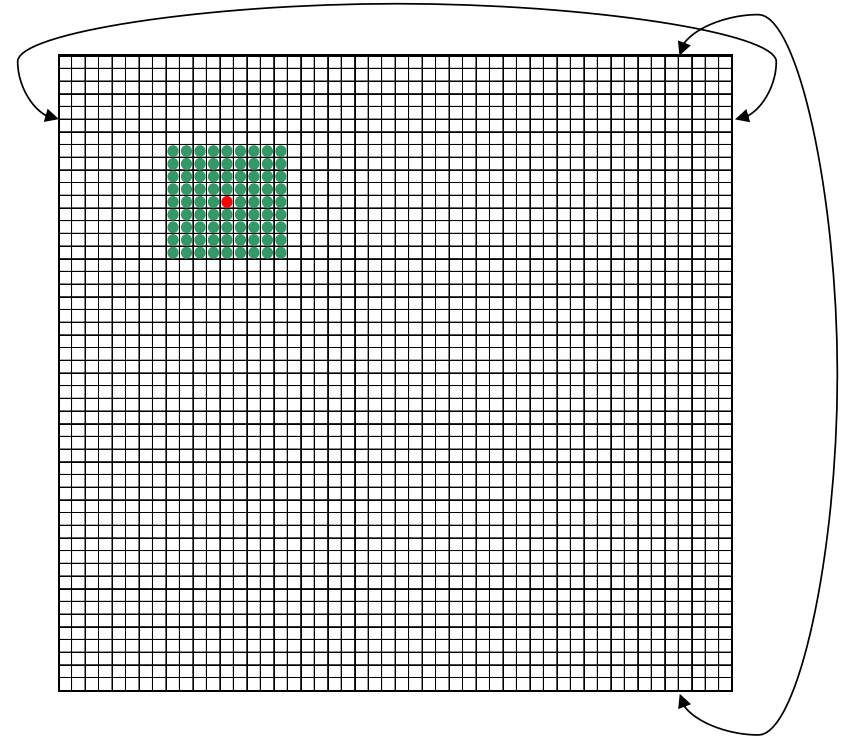

Figure 5: 50x50 Toroidal Lattice Social Network Structure Showing a Moore Neighborhood of Radius Four

\subsection{Simulation Process Details}

In the neighborhood simulation, actors possess the attitudes and behavior, and pairs of neighbors may be friends. They influence each other by their attitudes and behavior while developing friendships and possibly moving out of the neighborhood. All of these influences are regulated by parameters.

Each iteration of the simulation is nominally one year, during which each agent's status is stochastically updated in fixed order. There is a fixed order for the update of attributes of an agent:

1. Moving out of the neighborhood

2. Building friendships (which accumulate)

3. Deciding whether to re-evaluate one's occupation

4. Updating attitude of efficacy

First it is determined whether the agent will leave based on the tenure and stability-related calculations of the probability of leaving detailed above. As the analysis of the data in Browning, Feinberg, and Dietz treats neighborhood instability as exogenous, the probability of leaving in this model does not depend on the crime rate. [In further research it may be worthwhile to consider a reciprocal relationship between crime rates and rates of moving, however.] If the agent leaves, it is replaced with a new agent. Otherwise the agent is updated.

A new agent is given a criminal status and propensity according to the probability utilized in the initialization of criminal status, and given values of baseline efficacy, friendship probability, and friendship status according to the initialization procedure. The starting value of tenure is one year.

An agent who does not move out of the neighborhood is influenced by the efficacy of both friends and non-friends in his Moore neighborhood, but according to separate coefficients. If the other is a criminal, the effective efficacy is set at a parameter; otherwise the effective efficacy is the actual efficacy. For both classes of Moore neighbors, the influence of the other on own efficacy is on a per-agent basis, making influence of the two categories proportional to their numbers divided by the total number of Moore neighbors. 


\section{Wilcox}

The latent probability of becoming a criminal is the $\exp (x) /(1+\exp (x))$ function of the sum of the agent's criminal propensity and products of coefficient parameters with the efficacies of friends and nonfriends in the agent's Moore neighborhood as well as the crime rates among friends and globally. In equations, we first apply the social and economic influences using the formula,

Defining as the Moore neighborhood of and as the set of Moore neighbors who are not friends of ,

the equation for set is similar except that the summation is performed over the , and

Note that another possible choice for these formulas would be to divide by the size of the sets over which the summations are performed, if nonempty. Also, the economic limitation due to the possibility of saturation in the neighborhood is calculated using the formula,

Then we transform

into the latent criminal probability as follows:

If a Bernoulli trial against the criminal status update probability is successful, criminal status is updated according to the latent probability.

Friendship cumulatively increases, and new friends are added from the Moore neighborhood according to the friendship creation probability. Tenure is incremented by one each iteration. Efficacy is based on the baseline efficacy for the agent calculated at initialization, plus the products of parameterized coefficients times the friend crime rate, the friend efficacy, and tenure.

\section{SIMULATION CALIBRATION METHODOLOGY}

Browning, Feinberg, and Dietz report a correlation matrix of the data analyzed in their regression analyses. Preparatory to calibration (parameter estimation) with the method in Wilcox (2005), stochastic optimization iterations (Spall 2003), or the method of simulated moments (McFadden 1989; McFadden and Ruud 1994; Stern 1997), comparable results from the model using a wide range of inputs are compared with the reported correlations in order to verify that the simulation results bracket the target data points. 


\section{Wilcox}

Here we take a subset of the correlations reported by Browning, Feinberg, and Dietz, and desire to estimate from them the structural parameters of the agent-based model. The correlations reported by Browning, Feinberg, and Dietz (2004) and utilized in this paper are presented in Table 1. The correlations highlighted in orange are the exogenous variables. The presence of the four-year lag of the crime rate gives us a reading on the level of consistency over time possessed by the phenomenon of crime, which is important with respect to the economic literature, as the level of instability has been the difficult part to explain. The means of the variables are not given. Many of the means and standard deviations are meaningless, as they are empirical Bayes residuals or attitude measures.

As survey estimates and the outcome of empirical Bayes purification, many of the subject variables are subject to estimation error themselves. This creates the problem of unmodeled measurement error in the predictor variables, which causes bias in the regression estimates and lowers the correlation estimates from their actual population values. Further refinement of the analysis would include adjusting the published correlations based on the estimates of the reliability of predictor measures reported by Browning, Feinberg, and Dietz (2004) and discussed in related papers.

Table 1: Data correlations presented by Browning et al (2004), from which they calculate their multiple regression results. These are the targets for simulation calibration using an agent-based model. The correlations highlighted in orange are exogenous to the model, while the others are created from batches of model runs that have the same parameter values.

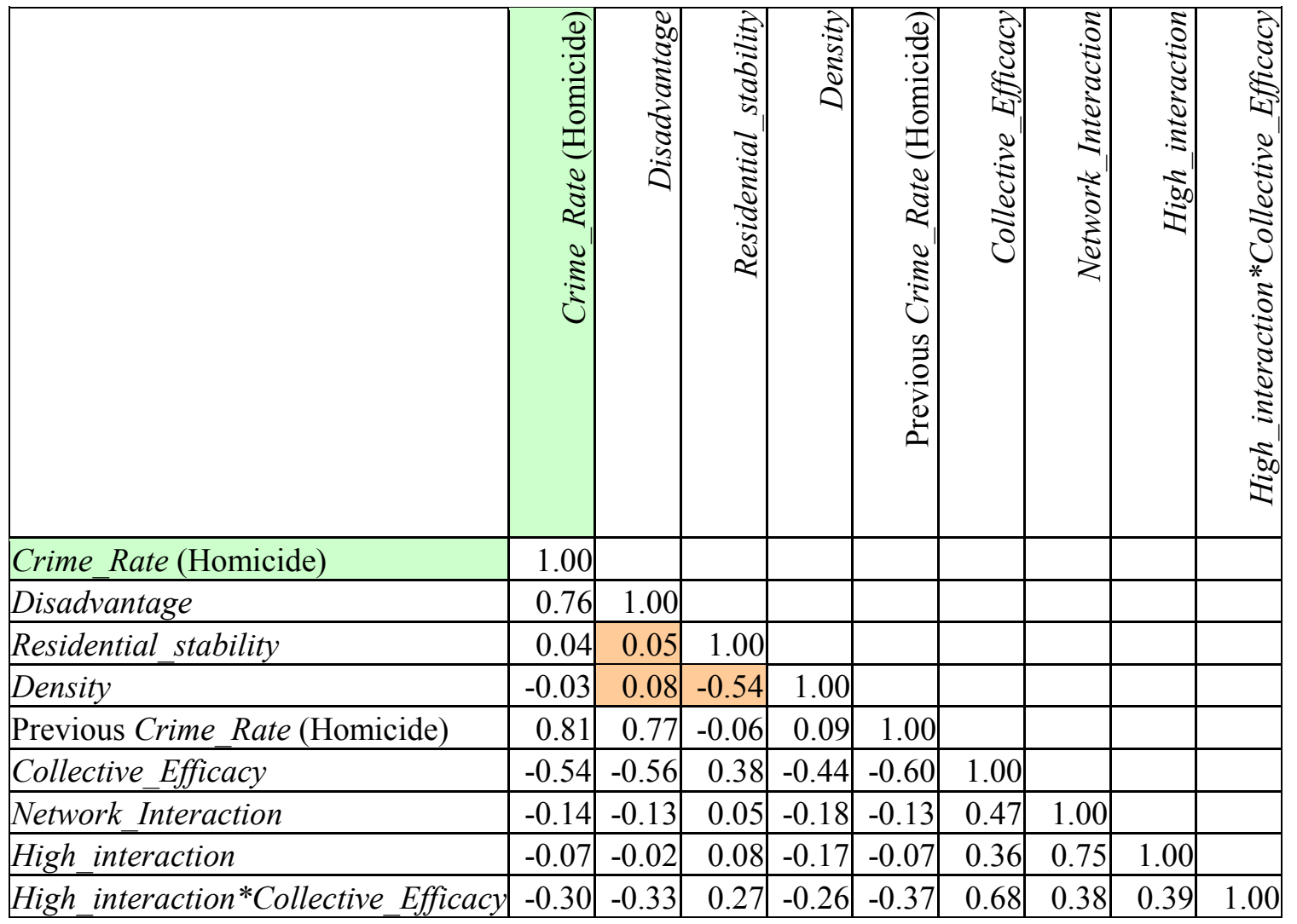

\section{DATA COLLECTION AND INITIAL ANALYSIS}

Ecological data is collected from all 2500 persons in the simulated neighborhood. After initialization, the model is executed for 20 iterations prior to the collection of the lagged log odds of the crime rate, given 
by the formula, $\log ($ CrimeRate / $(1$ - CrimeRate $))$, and then executed for 4 more iterations prior to the collection of the rest of the data. Residential stability is collected as the mean of tenure (in simulation iterations). Network interaction is the mean of the friendship status indicator variable. Efficacy is also the mean of this variable before its adjustment for criminal status. The log odds of the crime rate is estimated using a numeric accommodation for the possibility of zero crime rates. The agent-based model in Wilcox (2005) was re-implemented in Java for speed.

Correlations of interest were calculated from batches of 30 independent model runs and used as data for the later method of simulated moments analysis. To achieve this, variables were calculated to support the interaction effects in the regression analysis reported by Browning, Feinberg, and Dietz. From the network interaction variable, the $70^{\text {th }}$ percentile was calculated in order to create the indicator for high network interaction and thus its product with collective efficacy.

The initial experimental design was to uniformly generate the simulation parameters within broad limits, respecting the theoretical boundaries at zero. From the batches of runs, correlations to match those in Browning, Feinberg, and Dietz were computed and displayed pair-wise in scatter plots along with the original data. As this highlighted ways in which the model did not fit the data, tweaking was performed to see if the lack of fit could be remedied using adjustments such as adding parameters or making minor and not-so-minor changes to the modeling of social influence.

Figure 6 shows four scatter plots in which the published correlations are particularly far from the simulated correlations over a broad range of parameters. Notice that some output correlation measures are associated with others in unusual-looking ways, while other pairs look to the eye like a correlated bivariate normal random variables. In the lower left-hand side of the figure, the baseline model's correlation between the network interaction dummy variable $(X X 33)$ and residential stability is fairly high, while the data reported by Browning, Feinberg, and Dietz has a very low correlation. Also, the correlation between collective efficacy and neighborhood disadvantage is in the neighborhood of zero in the simulation results, but negative in Browning, Feinberg, and Dietz. Looking at the upper right, Browning, Feinberg, and Dietz report values for the correlations of $X X 33$ with residential density as well as that between network interaction and density that are far from the range of values coming from the simulation. In the lower right, we see that the simulated values of two correlations (network interaction with collective efficacy and the interaction variable, $X X 34$, with residential stability) form a wedge shape that excludes the reported point. Moreover, the points in the scatter plot on the upper left also seem to nestle up against a wall, on the other side of which is the published correlation.

Figure 7 shows the four scatter plots of Figure 6 in a version of the tweaked model in which progress may have been made in obtaining output correlations that bracket the data points. The simulated range of correlations is closer to the published values for $X X 33$ with residential stability seen in the vertical axis of the plot on the lower left, while the 'wall' seen in the plot on the upper left is more pronounced. In both the upper left and lower right, we see how the two dimensional character of the scatter plots reveal the inability to match correlations that would not be revealed by looking at them in a univariate manner.

\section{CONCLUSIONS}

Employing an agent-based simulation as a replacement for theory increases the precision of one's arguments, but at a price. With the simulation, the domain of modeling concern increases as one examines the arguments and aligns the theory. Because of the increased rigor of this process, the need for elaboration ensues beyond what was apparent from the prose expression of the theory. While Browning, Feinberg, and Dietz were able to test their theory by looking at a single interaction effect in a regression model, calibrating a simulation model to the correlation matrix employed in the regression model estimation led to any one of the correlations sufficing to invalidate the simulation. By instantiating social theory as a formal system, any issues with the reasoning linking theory to hypotheses are more easily laid bare than with a prose description of the same social system.

Some of the difficulty in replicating the published correlations may relate to the performance of secondary data analysis. The manner in which the measures in Browning, Feinberg, and Dietz were purified 


\section{Wilcox}

may be an issue, as may be the fact that they are based on samples, which leads to an errors-in-variables problem and corresponding deflation of correlation measures. Also, the use of normalized measures, while not creating a problem for multiple regression models, results in loss of information that can be remedied by using the original data.

In line with the tradition of network-based influence models, the current model is based on the assumption that social influence is network-based. Moreover, friendship development is driven by length of time in the neighborhood, which relates to its instability. However, a less conventional approach to consider in further research would be to take into account the power of weak ties (Granovetter, 1973) and model neighborhood efficacy as a reputation that is built up as a result of experience with proactive neighbors, rather than as an outcome of social influence along strong ties.
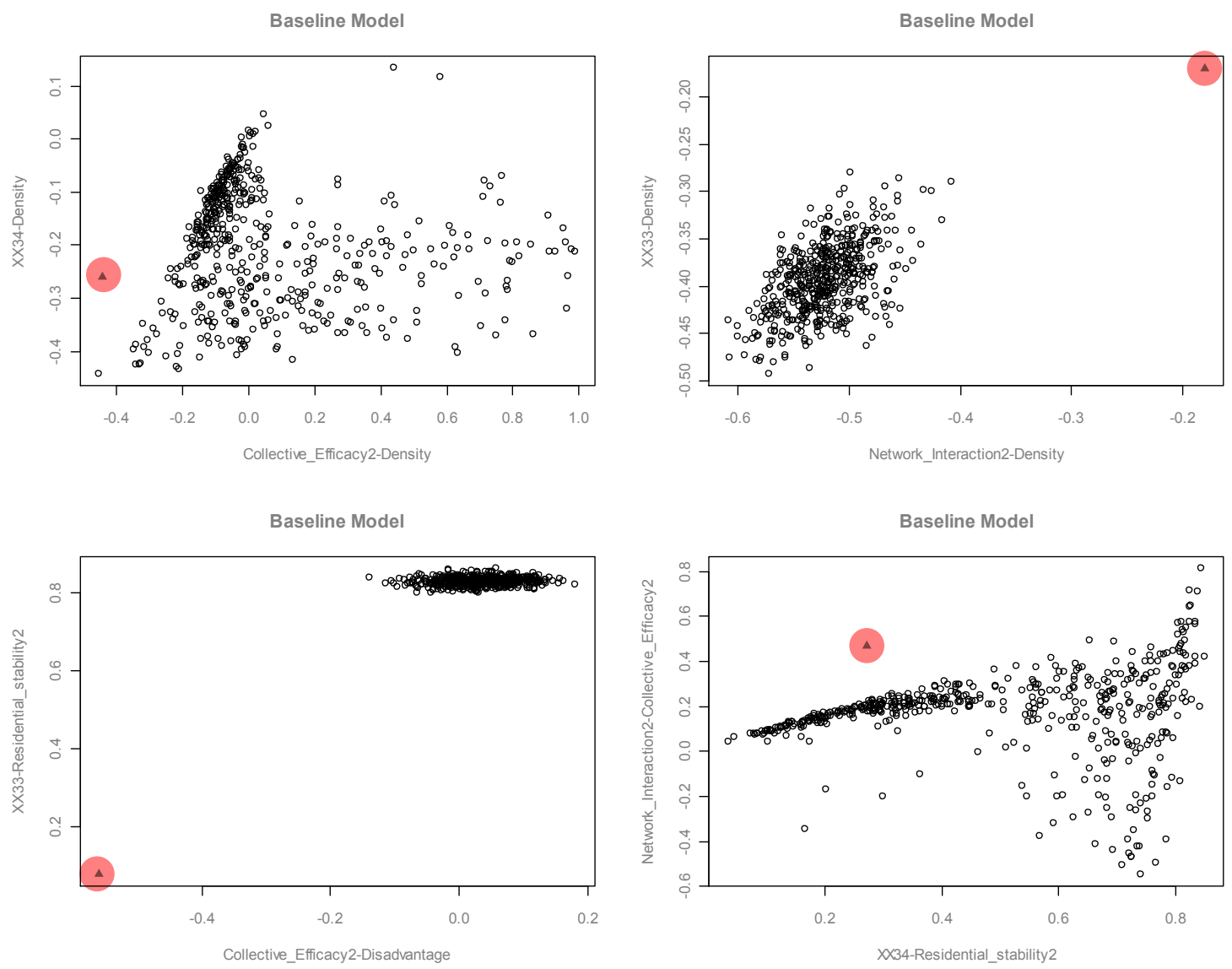

Figure 6: Selected scatter plot results with the baseline model using a wide range of parameter settings, compared to the published correlations (highlighted in red). Looking at these plots, it is clear that the simulation model does not fit the correlations reported by Browning, Feinberg, and Dietz (2004). 

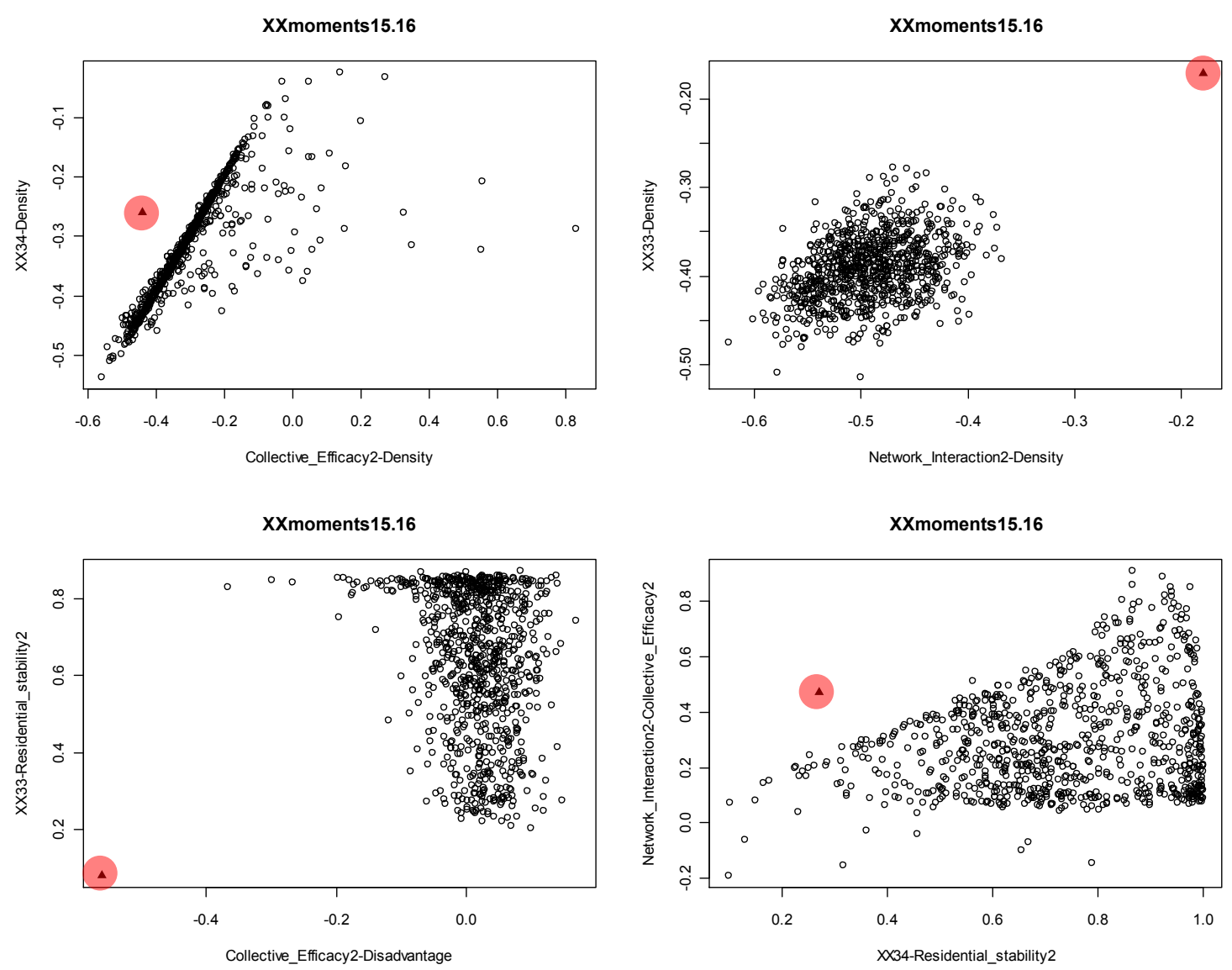

Figure 7: Scatter plot results with the modified model using a wide range of parameter settings, compared to the published correlations. The simulation model still does not fit the reported correlations.

\section{REFERENCES}

Becker, G. S. 1968. “Crime and Punishment: An Economic Approach.” The Journal of Political Economy 76(2):169-217.

Browning, C. R., S. L. Feinberg, and R. Dietz. 2004. "The Paradox of Social Organization.” Social Forces 83(2):503-534.

Calvó-Armengol, A. \& Y. Zenou. 2004. "Social Networks And Crime Decisions: The Role Of Social Structure In Facilitating Delinquent Behavior." International Economic Review, 45(3):939-958.

Crane, J. 1991. "The Epidemic Theory of Ghettos and Neighborhood Effects on Dropping Out and Teenage Childbearing." American Journal of Sociology 96:1226-59.

Glaeser, E. L. and B. Sacerdote. 1999. "Why Is There More Crime in Cities?" The Journal of Political Economy 107(6), Part 2: Symposium on the Economic Analysis of Social Behavior in Honor of Gary S. Becker (Dec., 1999): S225-S258.

Granovetter, M. S. 1973. "The Strength of Weak Ties.” American Journal of Sociology 78(6):1360-1380.

Harrison, J. R. and G. R. Carroll. 2002. "The Dynamics of Cultural Influence Networks." Computational \& Mathematical Organization Theory, 8(1): 5-30.

Kasarda, J. D., and M. Janowitz. 1974. "Community Attachment in Mass Society." American Sociological Review 39:328-39.

Kornhauser, R. R.. 1978. Social Sources of Delinquency. University of Chicago Press. 
Lustick, I. S. 2000. “Agent-based Modeling of Collective Identity: Testing Constructivist Theory.” Journal of Artificial Societies and Social Simulation, Vol. 3(1). http://jasss.soc.surrey.ac.uk/3/1/1.html.

Lustick, I. S. 2002. "PS-I: A User-Friendly Agent-Based Modeling Platform for Testing theories of Political Identity and Political Stability." Journal of Artificial Societies and Social Simulation 5(3). http://jasss.soc.surrey.ac.uk/5/3/7.html.

Mahoney, J. 2004. "Revisiting General Theory in Historical Sociology." Social Forces, 83(2):459-489.

Makowsky, M. 2006. "An Agent-Based Model of Mortality Shocks, Intergenerational Effects, and Urban Crime." Journal of Artificial Societies and Social Simulation 9(2):7. http://jasss.soc.surrey.ac.uk/9/2/7.html.

McFadden, D. 1989. "A Method of Simulated Moments For Estimation of Discrete Response Models Without Numerical Integration." Econometrica 57(5):995-1026.

McFadden, D. \& Ruud, P. A, 1994. "Estimation by Simulation." The Review of Economics and Statistics 76(4): 591-608.

Schelling, T. S. 1971. "Dynamic Models of Segregation.” Journal of Mathematical Sociology 1:143-186.

Spall, J. C. 2003. Introduction to Stochastic Search and Optimization. Hoboken, New Jersey: Wiley.

Stern, Steven. 1997. "Simulation-Based Estimation." Journal of Economic Literature 35 (December):2006-2039.

Troitzsch, Klaus G. 2008. "The Garbage Can Model of Organisational Behaviour: A Theoretical Reconstruction of Some of Its Variants." Simulation Modeling Practice and Theory 16:218-230.

Whyte, W. F. 1937. Street-Corner Society. Chicago: University of Chicago Press.

Wilcox, S. P. 2005. "Agent-Based Models as Quantitative Sociological Methodology." In Proceedings of the Agent 2005 Conference on Generative Social Processes, Models, and Mechanisms, October 13-15, 2005, ANL/DIS-06-1, Edited by C. M. Macal, M. J. North, and D. Sallach. Chicago, Illinois: Argonne National Laboratory.

Wilson, W. J. 1996. When Work Disappears. Knopf.

\section{AUTHOR BIOGRAPHY}

STEVEN P. WILCOX is a defense analyst with Serco Inc, and is currently Immediate Past President of the Washington, D.C.-area chapter of INFORMS. After receiving a Masters in Sociology and his Ph.D. in Operations Research from the University of North Carolina at Chapel Hill, he joined GRC International to develop military manpower models for the U.S. Army, then worked for Northrop Grumman in Arlington, Virginia, after which he went to Serco to develop manpower models for the U.S. Navy. In the process, he acquired an M.B.A. and an M.S. in Statistical Science from George Mason University and is now working on a Ph.D. there, this time in Computational Social Science. 